Results In the study period, there were 6626 OHCAs attended by Prague EMS, of which 121 met the criteria of $\mathrm{CH}-\mathrm{OHCA}$ (1.8\%, $0.78 \mathrm{CH}-\mathrm{OHCA}$ per 100.000 inhabitants and year). The majority of $\mathrm{CH}-\mathrm{OHCA}$ occurred in age groups $0-0.99(35 ; 28.9 \% ; \mathrm{p}<0.05)$ and $1-1.99(26 ; 21.5 \% \mathrm{p}<0,05)$. In no other age group the number of $\mathrm{CH}-\mathrm{OHCA}$ exceeded 9 (n/s). No CH-OHCA was witnessed by ambulance personnel. Layperson CPR was performed in 95 cases $(78,5 \%)$. The first captured rhythm was most often asystole $(96 ; 79.4 \%)$. Ventricular fibrillation (VF-first subgroup) as the first rhythm was present in 12 patients (10.0\%). The most common cause of $\mathrm{CH}-\mathrm{OHCA}$ was recorded as unknown (39\%). The overall survival rate from $\mathrm{CH}-\mathrm{OHCA}$ (with $\mathrm{CPC} 1-2$ ) was $10.7 \%$, in VF-first subgroup it was $41.7 \%$.

Conclusion $\mathrm{CH}-\mathrm{OHCA}$ is relatively rare event. The most vulnerable group is children under 2 years of age. Small children are most frequently found in asystole, while VF occurs more frequently in age over 15. Even though $\mathrm{CH}-\mathrm{OHCA}$ is uncommon in pre-hospital setting, everyone in the emergency service must be adequately trained for it.

Conflict of interest None

Funding None

\section{A CENTRALISED INTER-FACILITY PATIENT TRANSFER SERVICE IN SOUTHERN FINLAND -THE HELSINKI EXPERIENCE}

${ }^{1} \mathrm{~F}$ Stenius, ${ }^{2} \mathrm{P}$ Holmström, ${ }^{2} \mathrm{~J}$ Etelä, ${ }^{2} \mathrm{~K}$ Sarvikivi, ${ }^{1,2} \mathrm{~T}$ Puolakka*. ${ }^{1}$ Department of Anaesthesiology and Intensive Care Medicine, University of Helsinki; ${ }^{2}$ Emergency Medicine and Services, Helsinki University Hospital, Helsinki, Finland

\subsection{6/10.1136/bmjopen-2018-EMS.37}

Aim Inter-facility patient transfers form an important share of out-of-hospital patient transport. In many countries these transfers are commonly done by small ambulance companies and by the local emergency medical services. The aim of this observational study was to report the operation of the only centralised inter-facility transfer service in Finland.

Method Helsinki University Hospital patient transfer service is responsible of all inter-facility transfers in the region with 1.6 million inhabitants. During a three-month cohort (1.931.11.2016) all patients transferred by the transfer service ambulances were selected for the study. The patient reports were collected and quality deviations during transport were registered based on predetermined criteria.

Results In a total of 3034 transfers, lights and sirens were required in $73(2.4 \%)$ cases. In $45(1.5 \%)$ transfers the patient was accompanied by a physician and in 35 (1.1\%) a nurse from the referring hospital. The most common issues requiring treatment during transport were pain (numeric rating scale $\geq 4)$ in $191(6.3 \%)$ and hypoxemia in 119 (3.9\%) transfers. Moreover, 147 (4.8\%) patients carried microbes important for hospital hygiene. Quality deviations were registered in 338 transfers $(8.4 \%)$. The most common deviations were insufficient or missing written reporting in 244/338 cases (72.2\%). Problems were also encountered with patient monitoring and treatment in 80/338 transfers (23.7\%). Adverse events were registered during 16 transfers $(0.5 \%)$.

Conclusion In daily inter-facility transfer service operations, the need for lights and sirens was uncommon. Acute pain and hypoxemia were the most common issues to be addressed during transport. Adverse events were rare.

Conflict of interest None
Funding None

\section{HEART RATE VARIABILITY AND PRE-HOSPITAL RISK STRATIFICATION OF CHEST PAIN PATIENTS}

${ }^{1}$ Chu En Ting ${ }^{*},{ }^{2,3}$ Nan Liu, ${ }^{4}$ Zhi Xiong Koh, ${ }^{4}$ Dagang Guo, ${ }^{4}$ Janson Cheng Ji NG, ${ }^{4}$ Swee Han Lim, ${ }^{4,5}$ Marcus Eng Hock Ong. 'Duke-NUS Medical School, Singapore; ${ }^{2}$ Health Services Research Centre, Singapore Health Senvices, Singapore; ${ }^{3}$ Centre of Quantitative Medicine, Duke-NUS Medical School, Singapore; ${ }^{4}$ Department of Emergency Medicine, Singapore General Hospital, Singapore; ${ }^{5}$ Health Services and Systems Research, Duke-NUS Medical School, Singapore

\subsection{6/10.1136/bmjopen-2018-EMS.38}

Aim Assessment of chest pain patients often requires extensive investigations, while existing risk-scores are designed for use in Emergency Departments (ED). We aim to develop a risk score for chest pain patients in pre-hospital settings and ambulances by utilising heart rate variability (HRV), a rapid, noninvasive reflection of cardiac stress states.

Method This study analysed 902 chest pain cases presenting to Singapore General Hospital (SGH) ED between 2010 and 2015. The data was divided into a model derivation set (616 cases, $32 \%$ meeting outcomes) and a validation set (286 cases, $28.7 \%$ meeting outcomes). HRV Prediction Model (HRV-PM) for 30 day Major Adverse Cardiac Events (MACE) was derived using backwardstepwise logistic regression. HEART, TIMI and GRACE scores were used as comparators for the HRV-PM.

Results In the derivation set, $66.9 \%$ of patients were male, with a mean (SD) age of 60.71 (12.97). HRV-PM encompasses 9 parameters: $6 \mathrm{HRV}$ variables (triangular interpolation of normal-to-normal intervals, total power, very-low/low/highfrequency power, approximate entropy), and 3 ECG variables (ST-elevation, ST-depression, Q-wave). Validation of HRV-PM shows similar performance to HEART for 30 day MACE prediction with Area Under Receiver-Operating-Curve (AUC) of 0.737 (95\% CI: 0.673 to 0.800$)$ versus 0.739 (95\% CI: 0.679 to 0.799$)$. The addition of 1 set of troponin results in HRV-PM improves the AUC to 0.749 (95\% CI: 0.686 to 0.813), thereby outperforming HEART.

Conclusion HRV-PM is a simple, non-invasive test demonstrating comparable performance with HEART without need of blood-tests. We hope to validate and apply HRV-PM for assessment of chest pain patients by EMS in pre-hospital settings including ambulances.

Conflict of interest None

Funding None

\section{BYSTANDER CPR IN OUT-OF-HOSPITAL CARDIAC ARREST IN SINGAPORE: AGE AND OTHER PREDICTIVE FACTORS}

${ }^{1}$ Jasmine Lim $Y^{*}$, ${ }^{2}$ Win Wah, ${ }^{2}$ Nur Shahidah, ${ }^{2}$ Susan Yap, ${ }^{2}$ Pek Pin Pin, ${ }^{3}$ Yih Yng Ng, ${ }^{4}$ Benjamin SH Leong, ${ }^{5}$ Han Nee Gan, ${ }^{6}$ Desmond R Mao, ${ }^{7}$ Michael YC Chia, ${ }^{8}$ Si Oon Cheah, ${ }^{9}$ Lai Peng Tham, ${ }^{2,10}$ Marcus EH Ong. 'Duke-NUS Medical School, Singapore; ' 2 Department of Emergency Medicine, Singapore General Hospital, Singapore; ${ }^{3}$ Medical Department, Singapore Civil Defence Force, Singapore; ${ }^{4}$ Emergency Medicine Department, National University Hospital, Singapore; ${ }^{5}$ Accident and Emergency, Changi General Hospital, Singapore; ${ }^{6}$ Department of Acute and Emergency Care, Khoo Teck Puat Hospital, Singapore; ${ }^{7}$ Emergency Department, Tan Tock Seng Hospital, Singapore; ${ }^{8}$ Emergency Medicine Department, Ng Teng Fong General Hospital, Singapore; ${ }^{9}$ Children's Emergency, KK Women's and Children's Hospital, Singapore; ${ }^{10}$ Health Services and Systems Research, Duke-NUS Medical School, Singapore

10.1136/10.1136/bmjopen-2018-EMS.39 
Aim Multiple studies affirm that Bystander CPR (BCPR) improves outcomes in Out-of-hospital Cardiac Arrest (OHCA). This study investigates age and other factors in affecting BCPR rates in Singapore.

Method Data was extracted from the national cardiac arrest registry. OHCA cases in Singapore conveyed via emergency medical services from April 2010-December 2015 were included. Zipcodes of arrest location, census data from 20102015 and planning areas were used for geospatial analysis. Multivariate logistic regression was performed to investigate the association between patient demographics and pre-hospital data with BCPR.

Results 8955 cases were split into 3 groups: Paediatric (Age 0-10 years; $n=107$ ), Young (Age $11-65$ years; $n=3979$ ), Old (Age $>65$ years; $n=4869$ ). Compared to the Old, Paediatric and Young patients had higher rates of BCPR (OR 3.997, 95\% CI: 2.567 to 6.224 ; OR 1.133, 95\% CI: 1.027 to 1.249). Non-traumatic arrest (OR 2.550, 95\% CI: 1.743 to 3.731) and Witnessed arrest (OR 1.267, 95\% CI: 1.157 to 1.387), positively associated with BCPR, while arrest in residential location (OR 0.655 , 95\% CI: 0.441 to 0.973 ) negatively associated with BCPR. Of the OHCA in residential locations, patients in neighbourhoods with younger population were more likely to receive BCPR (OR 1.242, 95\% CI: 1.061 to 1.453$)$ compared to older neighbourhoods. There was no association with gender and major race groups, and univariate analysis showed no difference in BCPR rates between family bystanders and other layperson bystanders.

Conclusion Older patients, residential arrests and older neighbourhoods were associated with lower rates of BCPR. Public education and training programs can be targeted to these populations to improve BCPR rates.

Conflict of interest None

Funding This study was supported by grants from National Medical Research Council, Clinician Scientist Award, Singapore (NMRC/CSA/024/2010 and NMRC/CSA/0049/2013), Ministry of Health, Health Services Research Grant, Singapore (HSRG/0021/2012) and Duke-NUS Khoo Research Student Award (Duke-NUS-KRSA/2013/0001).

\section{THE LONG TERM EFFECTS OF A 3-DAY FIRST AID PROGRAMME FOR 7-14 YEARS OLD PRIMARY SCHOOL CHILDREN}

'B Banfai*, 'A Pandur, 'B Schiszler, 'B Radnai, ${ }^{2} \mathrm{H}$ Banfai-Csonka, '1 Betlehem. ' University of Pécs Faculty of Health Sciences Institute of Emergency Care and Health Pedagogy, Hungary; ${ }^{2}$ University of Pécs, Department of Emergency Medicine, Hungary

\subsection{6/10.1136/bmjopen-2018-EMS.40}

Aim Bystanders can play an important role in the event of sudden injury or illness. Our aim was to evaluate the long term effects of a 3 day first aid programme for all primary school age groups (7-14 years old).

Method This study was a 15 months follow-up of our previous investigation. ${ }^{1}$ Five-hundred twenty-four primary school children were involved in this study (288 girls and 236 boys). Measurements were made in the following topics: adult basic life support, using an automated external defibrillator, handling an unconscious patient, managing bleeding and calling the ambulance. Data collection was made with a self-made questionnaire and observation.

Results Knowledge and skills after 15 months were significantly higher in the majority of the measured topics than before the programme $(p<0.01)$. However, results were significantly worse than immediately and 4 months after programme $(p<0.01)$. Younger children overall remembered less well than older children $(\mathrm{p}<0.01)$. Approximately three-third of children could use an AED, know the emergency phone number and the half of them could perform correct recovery position at 15 months. Correct assessment of breathing was similar than in baseline.

Conclusion This study was the most comprehensive longitudinal study so far conducted on the effects of first aid (not only resuscitation) programme among primary school children. Participants could remember some aspects of first aid long term. However, forgetting after 15 months was significant so refreshing trainings would be mandatory annually

\section{REFERENCE}

1. Banfai B, Pek E, Pandur A, Csonka H, Betlehem J. 'The year of first aid': Effectiveness of a 3-day first aid programme for 7-14-year-old primary school children. Emerg Med J 2017;34:526-32.

Conflict of interest None

Funding None

\section{IS IT NECESSARY? - OPINIONS AND EXPERIENCES ABOUT FIRST AID EDUCATION IN CHILDHOOD - PILOT STUDY}

${ }^{1}$ B Banfai*, ' A Pandur, 'B Schiszler, 'B Radnai, ${ }^{2} \mathrm{H}$ Banfai-Csonka, ${ }^{1} \mathrm{~J}$ Betlehem. 'University of Pécs Faculty of Health Sciences Institute of Emergency Care and Health Pedagogy, Hungary; ${ }^{2}$ University of Pécs, Department of Emergency Medicine, Hungary

\subsection{6/10.1136/bmjopen-2018-EMS.41}

Aim Starting first aid education in early childhood can be an effective method of knowledge transfer. Our aim was to examine first aid education related opinions of kindergartenand primary school children, educators, and parents.

Method Eight-hundred seventy-one people (700 kindergartenand primary school children, 29 educators, 142 parents) were involved in our study. Opinions were measured with selfedited questionnaires, containing mainly open questions.

Results Based on the answers, the majority of children (90\%) enjoyed our programme, they would like to participate further first aid programmes in the future. General opinions of parents and educators about first aid education in childhood were mainly positive, but they also gave negative ones. The majority of participants $(>90 \%)$ had a positive opinion about our completed programme. ${ }^{1}$ Based on the opinions of parents and educators first aid education is recommended by health care professionals. Opinions were independent from gender $(p=0.302)$, age $(p=0.512)$, time of working with children $(\mathrm{p}=0.251)$.

Conclusion Children's opinion about the first aid programme were mainly positive. General opinions of parents and educators were different, but were positive related our programme. Based on this results opinions can change with concrete activities and can make first aid education more popular/acceptable by laypeople. 